\title{
CLAIRCITY PROJECT: CITIZEN-LED SCENARIOS TO IMPROVE AIR QUALITY IN EUROPEAN CITIES
}

\author{
ENDA HAYES ${ }^{1}$, ANDREW KING $^{2}$, ALASTAIR CALLUM ${ }^{2}$, BEN WILLIAMS $^{1}$, \\ KRIS VANHERLE ${ }^{3}$, CORRA BOUSHEL ${ }^{1,6}$, JO BARNES ${ }^{1}$, TIM CHATTERTON ${ }^{1}$, \\ HANS BOLSCHER ${ }^{4}$, EVA CSOBOD ${ }^{5}$, LAURA FOGG-ROGERS ${ }^{6}$, \\ STEPHAN SLINGERLAND ${ }^{4}$ \& JAMES LONGHURST ${ }^{1}$ \\ ${ }^{1}$ Air Quality Management Resource Centre, University of the West of England, UK \\ ${ }^{2}$ PlayWest, University of the West of England, UK \\ ${ }^{3}$ Transport Mobility Leuven, Leuven, Belgium \\ ${ }^{4}$ Trinomics BV, Rotterdam, The Netherlands \\ ${ }^{5}$ The Regional Environmental Centre for Central and Eastern Europe, Szentendre, Hungary \\ ${ }^{6}$ Science Communication Unit, University of the West of England, UK \\ ${ }^{7}$ Norwegian Institute of Air Research, Lillestrom, Norway \\ ${ }^{8}$ Centre for Environmental and Marine Studies, University of Aveiro, Aveiro, Portugal
}

\begin{abstract}
Air pollution has as significant impact on the health of European citizens, particularly in urban areas, and was responsible for more than 400,000 premature deaths in Europe in 2014. It is within our cities where this public health impact is most acute as high population densities and high ambient concentrations result in an increased risk of exposure. This health impact is further exacerbated as air pollution interacts with other social determinants of health creating a disproportionate risk and burden. Traditional top-down government policy development and consultation, married with a very technocentric view of air pollution sources and solutions, has resulted in a general apathy among citizens towards the problem of air pollution and subsequently a lack of ownership of the solutions. The ClairCity Project aims to create a major shift in public understanding towards the causes of poor air quality, inviting citizens to give their opinions on air pollution and carbon reduction policies to shape the cities of the future. By putting citizens' behaviour and activities at the heart of policy making for air quality and carbon management, ClairCity has utilised a suite of innovative toolkits for enhanced quantification and citizen engagement (such as surveys, workshops, online games and apps) to assess the public acceptability of specific policies and also to enable a bottom-up, citizen-led scenario creation process whereby citizens are empowered to visualise clean, low carbon, healthy futures for their city. Keywords: air pollution, citizens, carbon, public engagement, games, scenarios, cities.
\end{abstract}

\section{INTRODUCTION}

Cities currently account for only $1 \%$ of the earth's surface but half of the world's population, $75 \%$ of global primary energy demand and $50-60 \%$ of global energy-related greenhouse gases (rising to $80 \%$ when indirect emissions are included) [1]. Up to a third of Europeans living in cities are exposed to air pollutant levels exceeding EU air quality standards with approximately $90 \%$ exposed to the WHO's more stringent guidelines [2]. Air pollution from EU industry alone is estimated to cost society $€ 59-€ 189$ billion in 2012 [3]. Future projections indicate that $70 \%$ of the global population in 2050 will be living in urban areas while cities, industry and commercial activities will require the majority of the forecasted $40 \%$ increase in world energy demand in 2020 [4]. These impacts cannot be sustained.

The complex links, both direct and indirect, between people's day-to-day activities and the collective demands that city populations put on local and global environments, particularly through poor air quality and increased carbon emissions, illustrates how the scope of the challenge extends beyond city geopolitical boundaries and reflects the need for long-term pathways to a low carbon, clean air future. 
A lack of civic engagement to date in the air quality debate lies partially in the absence of "people" in the models and scenarios used to estimate and predict air pollution concentrations. The modelling of emissions sources, not the human activities that result in them, leads to a bias in policy that focuses on mitigating emissions through technological change rather than through changing individual and societal behaviour [5]. In turn, this arguably leads to an over-reliance on technological innovation not social innovation. Recent high-profile cases such as the 2015 Volkswagen diesel emissions scandal and subsequent debate on real-world versus test cycle emissions brought to the fore that overreliance on technology alone would not solve our pollution problems. Traditional source apportionment approaches have previously considered the "technology" that has been responsible for creating the emissions (e.g. cars, heavy goods vehicles, combustion plant, etc.). The ClairCity project (www.claircity.eu) (EU Ref: 689289) goes beyond this to provide a new perspective and a new geography of pollution based instead on "activities, behaviour and practices" which will clearly allow the connection to be made between pollution and behaviour, and link these to the various practices that constitute everyday life within our cities. In other words, air pollution and carbon management are no longer to be addressed as separate and rather technical policy topics, but to be regarded as part of wider concerns of city inhabitants about their quality of life and healthy futures.

Current practices also tend to target the manifestations of problems rather than the cause (e.g. by focussing on air pollution hotspots, and on transport rather than the behaviour and activities that generate transport demand) [5]. Existing approaches to air quality and carbon management scenarios and policy development are designed to project forward from our current city baselines to achieve reductions in future years. This results in our cities developing into "what we end up with" rather than "what we want". To address this issue, the central concept of innovative, immersive and quantifiable citizen and stakeholder engagement through the ClairCity project in six European pilot cities (Bristol, United Kingdom; Amsterdam, Netherlands; Ljubljana, Slovenia; Sosnowiec, Poland; Aveiro, Portugal; and Liguria, Italy), will allow city citizens to create bottom-up future scenarios and backcast from their own visions of a future desirable city in order to work out what is necessary to do to achieve "what we want".

This paper explores the application of various engagement methods, specifically the ClairCity Skylines Game, to provide platforms for engagement and to generate the evidence need to enable citizens and other stakeholders to create bottom-up, citizen-led scenarios and to discuss the role of air quality and carbon policies for citizens' health, well-being and integral future quality of life.

\section{METHODOLOGY}

ClairCity has a number of interconnected work packages (WP) which consider the application of the latest social science thinking (WP3); immersive citizen engagement through Games and Apps as engagement tools (WP4); high-quality modelling of activities, emissions, concentrations and health outcomes (WP5); and scenario development and policy recommendation packages for inclusive future citizen-led air quality and carbon policies in cities and at national and EU levels (WP6 and WP7). The final deliverable for each city is a democratically generated, practical, quantified and applicable city policy package for a cleaner, low carbon, health future.

The schematic below describes the ClairCity approach in practical terms, illustrating the steps and engagement points between ClairCity and city citizens and stakeholders. The ClairCity Toolkit and Method has three distinct phases with each work package engaging with the city at different points and in different ways. These are described below: 
1. Phase 1: Evidence - work closely with the city, key stakeholder and citizens to collate the evidence to inform the behaviour-activities models and undertake a baseline policy analysis.

2. Phase 2: Engagement - undertake numerous engagement methods such as Delphi, Mutual Learning Workshop and the ClairCity Skylines Game to inform the development of citizen-led policy preferences and pathways.

3. Phase 3: Results - develop and quantify the citizen-led scenarios from the Delphi/ Game / Mutual Learning Workshop and present these back to the city as a bespoke city policy packages providing each city with a roadmap towards a clean-air lowcarbon healthy future.

The scenario development process is primarily driven by three citizen and stakeholder engagement activities delivered across all six case study cities - the Delphi process, the Mutual Learning Workshops and the ClairCity Skylines Game. This paper will focus on the design, application and early results of the ClairCity Skylines Game but introductory information on the other two methods are provided for context.

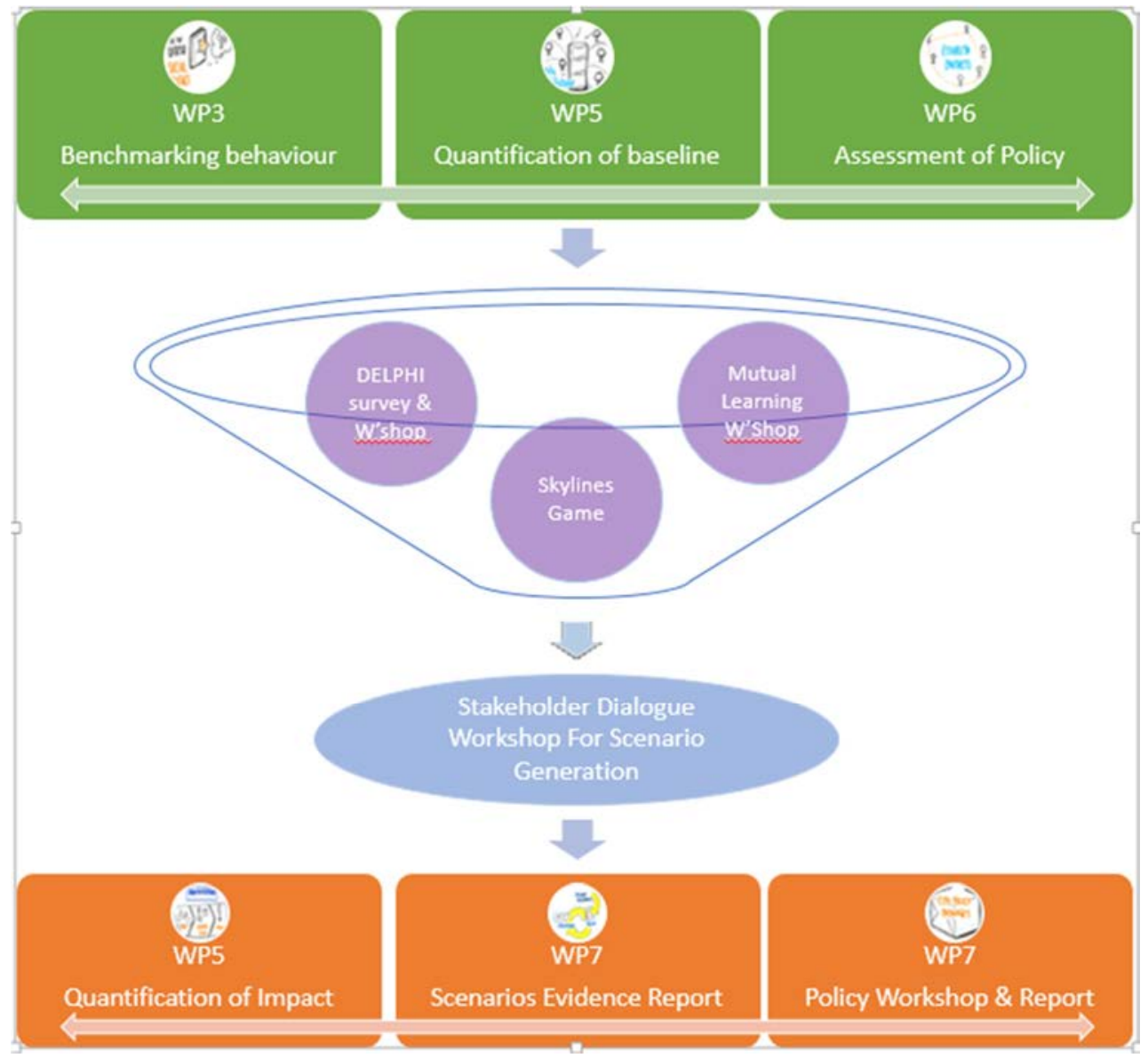

Figure 1: A simple illustration of the ClairCity process. 


\subsection{Citizen and stakeholder delphi survey and workshops}

Citizen and Stakeholder Delphi Survey and Workshops is designed to engage city citizens and stakeholder to generate qualitative and semi-quantitative evidence to help establish the citizen's attitude in terms of what they "like", "dislike" and "what would they change" in their city. Through two rounds of surveys and a workshop in a quasi-Delphi process, we use the city citizens as an "expert" panel to identify and seek consensus on:

1. The main sources and barriers to behaviour change (e.g. car dependant transport, cost driven solid fuel burning, lack of green space etc.);

2. The positive policies that might exist to enable and encourage behaviour change (e.g. safe and secure cycling infrastructure such as dedicated cycle lanes and bicycle storage).

The Delphi process also provides an engagement platform for both blue-sky and information driven thinking on pathways for what a low-carbon clean air-healthy future for their city looks like.

\subsection{Mutual learning workshop}

The Mutual Learning Workshop is designed to bring citizens and stakeholders together to better understand the specific challenges and opportunities that their city currently offers and encourage them to move towards reducing pollution and carbon emissions, improving air quality and decreasing health risks. This engagement platform brings citizens, community groups, transport operators, businesses, health professionals, air quality practitioners, city employees and elected officials together to share and discuss the health risk factors of the changing city environment, explore available solutions and map policies and pathways forward for a clean air, low carbon, healthy future.

\subsection{ClairCity Skylines Game}

The ClairCity Skylines Game creates a serious game experience to actively engage city citizens and stakeholders with an enhanced understanding of the air quality, carbon and health issues within cities. The Skylines Game allows the players (citizens and key stakeholders) to become the Mayor of ClairCity and gives them the power to augment a stylised "living city" that reflects data and evidence seeded from other ClairCity activities such as the Delphi process (WP4), Mutual Learning Workshop (WP4) and Baseline Policy Assessment of each city (WP6). The local design and feel of the Skylines Game (e.g. the game platform for Bristol is styled to look like Bristol) allows the player to feel comfortable and connected with their gaming environment. The scientifically robust game engine makes it possible to visualise when, where and why any player or group of players made key decisions and behavioural choices that led to the overall success or failure of their pathway. Unlike "gamified" simulations the game retains players, through offering the unique opportunity to replay and try-out alternative strategies and behaviours. This iterative approach significantly reinforces the impact of the experience, the message and the required real-world action. The player is forced to question their pre-conceived notions and the emotional reality of their envisaged future against the reality of their own decisions and the trade-offs that they are willing to make to achieve their low carbon, clean air future. 


\subsubsection{ClairCity Skylines Game core mechanic}

The Skylines Game has a comprehensive policy library (the ClairCity Policy Library (CPL)) underpinned the "ideas" that are presented to the players. Each of the $>500$ environmentally positive and negative "ideas" in the CPL has been mined and adapted from:

1. Existing publicly available databases of city solutions such as the JOAQUIN Database (www.joaquin.eu) and the FAIRMODE Catalogue of Air Quality Measures (http://fairmode.jrc.ec.europa.eu/measure-catalogue/) etc.,

2. The results of the Delphi process, Mutual Learning Workshop and baseline policy assessments; and

3. The knowledge of the ClairCity consortium.

Each individual "idea" has been scored (+/- 10 points) for both the short and long term impacts against four key attributes - climate/carbon, air quality/health, citizen satisfaction/happiness and city economy. Each individual policy is also categorised at four levels:

1. Level 1: Source Sector - This includes sources such as transport, energy, industry, waste, agriculture etc

2. Level 2: Source Sub-Sectors - Each Level 1 category is further subdivided. For example, transport is sub-divided to include active travel, public transport, private cars, van/HGV, taxis, aviation, shipping etc

3. Level 3: Policy Type - each policy in the database is classified by policy type such as regulatory, financial incentive, technological improvement, information provision etc

4. Level 4: Responsible Authority - each policy in the database is classified by the responsible body ranging from the individual to the EU Commission.

Players have to provide some simple demographic data (e.g. age, gender and rate their knowledge of air pollution from "nothing" to "expert") that will allow the project to undertake case attribute analysis of the results in the future to allow for demographically targeted scenarios and policy development. Players move around their stylised city to recognisable landmarks where they are randomly presented "ideas" from the CPL and they must chose the "ideas" they like. Chosen "ideas" are then promoted to "policies" by the player every five years with the aim to improve air quality, carbon and health in the city while maintaining citizen satisfaction and the city economy. At the end of each play (win or fail) the "ideas" and "policies" are recorded and can then be assessed to understand the public perception and acceptability of specific policies and policy areas thereby allowing ClairCity to "crowd-source" potential citizen-led policy pathways and scenarios.

To enhance the playability of the game the attribute scores (i.e. how well or how bad a player is doing against each of the four attributes) is linked to the game world effects so that the game world can either decay or regenerate. The attributes, game area and visual effect are outlined in Table 1 below.

\subsubsection{ClairCity Skylines Game promotion and evaluation}

The success of the Skylines Game is directly linked to the promotional efforts to engage players and generate awareness. Efforts include: commuter pop-ups in train and bus stations; promotion through social media; promotion by local networks and community groups; engagement with events with large footfall such as walking and running festivals, science centre days; and local media news include launch events with the Bristol Mayor. 
Table 1: Skylines Game attribute, game area and visual effect.

\begin{tabular}{|l|l|l|}
\hline Attribute & Game area & Visual effect \\
\hline Climate/carbon & Green space & Smog \\
\hline Air quality/health & Hospital & Citizen sickness \\
\hline Citizen satisfaction & Tourist area & Citizen happiness \\
\hline City economy & Bank & Building/city dereliction \\
\hline
\end{tabular}

Additionally, the Skylines Game has an evaluation step to better understand the player experience, provide feedback on the game design and playability and recommendations for enhancements for future iterations which will be rolled out across the other five ClairCity case study cities and regions.

\section{RESULTS}

To date (May 2018) and within the first month of the Skylines Game launch, the Bristol version of the Skylines Game has been downloaded $>750$ times with a play rate of 3.2 players per download (i.e. approximately 2,400 individual play sessions). During these plays, $>37,500$ "ideas" were presented, $>18,000$ individual "ideas" where collected for future consideration and $>10,000$ ideas were enacted into "policies". When exploring the enacted policies in more detail we see a shallow but wide variety of preferred policies being chosen by the Bristol players (see Fig. 2 and Fig. 3 below). These specific policies, while interesting in their own right, also illustrate the main policies areas of importance for citizens in that city. Additionally, this evidence is not viewed in isolation but also cross-referenced against the findings of the Delphi process and the Mutual Learning Workshop. Indicative results for Bristol point to three main areas of concern (Fig. 2) but when we looks at the "Top 10" enacted policies we see a clear preference for transport and land-use policies (Fig. 3). Indicative policy areas and policy preferences include:

- Transport (60.1\%): Citizens/players enacted policies addressed active travel (26\%) and public transport (25\%).

- Energy/Other (39\%): Citizens/players enacted energy policies addressed energy alternatives such as renewables (22\%) and energy efficiency measures $(18 \%)$ and

- "Other" policies addressed green infrastructure / green space (17.2\%).
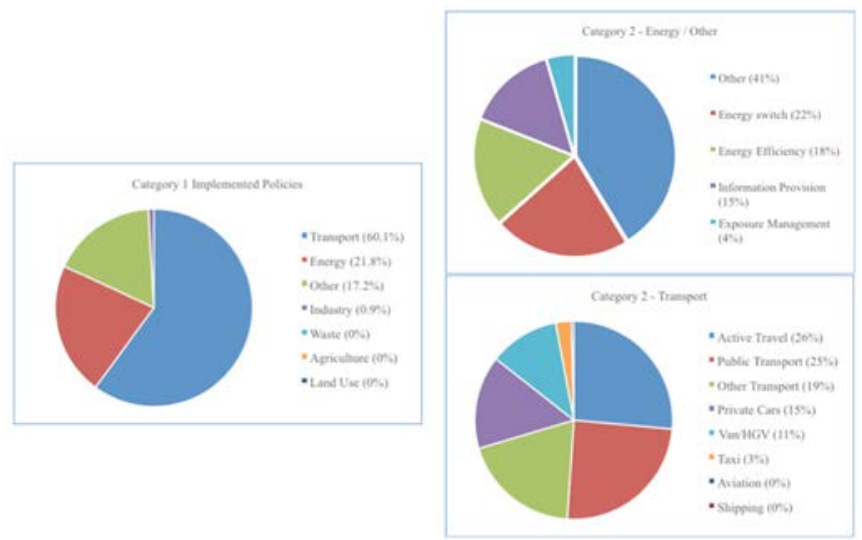

Figure 2: Indicative preferred policy categories from the Skyline Game in Bristol. 


Encourage gardens on rooftops
Fund development of cleaner delivery vans
Better road infrastructure for bikes, pedestrians and
motorbikes

Figure 3: Example "Top 10" citizen policy choices from the Skyline Game in Bristol.

\section{DISCUSSION}

Two decades of established emissions inventories and evolving modelling practices across the European Union have only taken air quality management and carbon reduction strategies so far. It can be argued that this is because the policy and methodologies used have, for a number of reasons, led us towards attempts to reduce emissions predominantly through technical measures, and away from changing the way our societies and cities operate and function. Atmospheric pollution is largely a consequence of society's use of energy whether it is for home heating and cooking, personal mobility, employment, industrial production etc. However, energy is not used for its own sake but as part of accomplishing social practices at home, at work and in moving around. Therefore, it can be argued that energy, and by direct association pollution, is an outcome of the social, infrastructural and institutional ordering of what people do. Our future cities will be unlike what they are today. Therefore, to truly understand how to mitigate air pollution and reduce carbon in our cities we need to ask what our cities use energy for and understand how end-uses of energy are changing.

The current state-of-the-art social science thinking on behaviour (including the recent social practice "turn" (e.g. Schatztki et al. [6], Shove et al. [7]) and Socio-Technical Systems and Transitions (e.g. Geels [8], [9]) are central to the ClairCity project to enable a step-change in how behaviour is accounted for in air quality and carbon management, not just by making it a central theme of analysis, but by also linking both social and individual orientated approaches to understanding and modelling behaviour [10]. While social-technical systems thinking and social practice theory are already being applied widely to carbon management, 
predominantly in the context of energy systems, it is yet to be applied to the challenge of air quality management and health outcomes, particularly at the level of city policies. By driving the subject area forward from a standpoint of behaviour and engagement, the conventional approaches to air quality management can be challenged by redefining the terms on which quantified analysis, policy making and scenario development is carried out.

By bringing a behaviour and social science dimension to the subject area, not only in terms of quantification but also in terms of citizen engagement, we can better represent how citizens see their own lives and can also make it more amenable to representing the significant societal changes and scnearios that are likely to occur between now and 2050. Innovative and immersive engagement platforms, such as the ClairCity Skylines Game, provide a platform for citizens to choose solutions while considering the personal impact but also to understand the aggregated impacts of their actions at the city level.

By putting citizens and their activities at the heart of the project, the quantification and engagement process goes beyond the traditional attempts to communicate air pollution in terms of ambient concentrations, whilst ensuring that the emphasis is placed on the actual health impacts of pollution on people themselves. In addition to making the information more salient, this will permit the project to make stronger links to the other negative, non-air quality/carbon, impacts related to relevant activities (such as noise, road safety, community separation etc.), and therefore place these issues and develop scenarios within the bigger picture of overall quality of life.

The next step requires bringing the results of the Delphi process, Skylines Game and Mutual Learning Workshop together to form the foundation evidence for future pathways and scenario construction. This evidence underpins a Stakeholder Dialogue Workshop to explore the variety of clean-air low-carbon healthy pathways chosen by the engagement participants/players and to examine and "crowd-source" a publicly acceptable consensus for these pathways in the short, medium and long term to 2050. The resulting "collective" pathway(s) will be used by the quantification work package (WP5) to model and quantify the future of a city, and by the Policy and Scenarios work packages (WP6 and WP7) to deliver a citizen-led, citizen-inclusive city relevant policy package.

\section{CONCLUSIONS}

The ClairCity project creates a new platform to stimulate discussion and engage citizens across Europe in a democratic debate about how their cities develop in a manner that protects the local and global environments and puts their health and well-being at the heart of policymaking. The latest social science thinking has been applied to the source apportion of air pollution emissions and concentrations, carbon emissions and health outcomes in order to attribute them not just by technology but by citizens' behaviours and daily activities. By putting people at the heart of both the problems and the solutions, the research has stimulated the public engagement necessary to tackle our challenging emissions problems through the development of a range of citizen-led future scenario and policy packages.

Through citizen-led future pathways, scenarios and people/behaviour targeted policies, the ClairCity project aims to improve cities' air quality (in line with EU Ambient Air Quality Directive (2008/50/EC) and National Emissions Ceiling Directive (2016/2284/EU)) and carbon footprints (aligned to specific city carbon reduction commitments), delivering subsequent health outcomes. Through the implementation of innovative and impactful toolkits and methodological approaches, ClairCity has given a voice to citizens and has enabled a new level of comprehensive and in-depth citizen and stakeholder engagement at the city scale, supported by scientifically robust quantitative and policy data and leading to a step change in democratic participation in environmental policymaking. 


\section{ACKNOWLEDGEMENT}

This project has received funding from the European Union's Horizon 2020 research and innovation programme under grant agreement No. 689289.

[1] UN-HABITAT for a better urban future. Urban Theme: Energy. https://unhabitat.org/urban-themes/energy/. Accessed on: 25 May 2018.

[2] European Environment Agency, Air Quality in Europe - 2017 Report, Technical Report No 13/2017, 2017.

[3] European Environment Agency, Cost of Air Pollution from European Industrial Facilities 2008-2012, Technical Report, No 20/2014, 2014.

[4] United Nations, World Population Prospects: The 2012 Revision, Methodology of the United Nations Population Estimates and Projection, ESA/P/WP.235, 2014.

[5] Chatterton, T., Air pollution: putting people at the heart of the issues, Environmental Scientist. Journal of the Institute of Environmental Science, 27(2), 2017.

[6] Schattzki, T.R., Cetina, K.K. \& von Savigny, E. (eds), The Practice Turn in Contemporary Theory, 44, 2003.

[7] Shove, E., Pantzar, M. \& Watson, M., The Dynamics of Social Practice: Everyday Life and How It Changes, SAGE: London, 2012.

[8] Geels, F.W., Technological Transitions as Evolutionary Reconfiguration Processes: A Multi-Level Perspective and a Case-Study, 31, pp. 1257-1274, 2002.

[9] Geels, F.W. A socio-technical analysis of low-carbon transitions: introducing the multi-level perspective into transport studies. Journal of Transport Geography, 24, pp. 471-482, 2012.

[10] Chatterton, T. \& Wilson, C., "The Four Dimensions of Behaviour" framework: a tool for characterising behaviours to help design better interventions. Transportation Planning and Technology, 37(1), pp. 38-61, 2014. 